\title{
The Simplicity in the Teaching of Human Anatomy for Artists
}

\author{
Bao Liu \\ Animation teaching and research section \\ Department of Art Design, School of Humanities \\ Yantai, Nanshan, University \\ Yantai, China, 265713 \\ e-mail: 598009865@qq.com
}

\begin{abstract}
After the brief introduction of "Simplicity" proposed by Gombrich and Arnheim, this paper makes an overall thinking and summaries on the methods of simplifying body parts in the teaching activities of human anatomy for artists. The simplicity in the teaching of human anatomy for artists is proposed in this paper, and the simplicity is classified into three categories, including alphabet, basic figures and symbols and daily items. It will help to find the rule of nature, which I hope could help researchers and students studying the teaching of human anatomy for artists.
\end{abstract}

Keywords-simplicity; the teaching of human anatomy for artists; symbol; shape

\section{INTRODUCTION}

In the teaching activities of human anatomy for artists, many people would spend lots of time studying one piece of muscle on, such as, its structure, start-stop position, function or memorizing its name. This will make the body more complicated so that people do not know how to begin the work and give people a feeling that there are lots of contents we need to acquire. In fact, many contents are about medical anatomy, which is of little practical use and would not be our focus. In the terms of research and teaching activities in human anatomy for artists, people have concluded two practice methods, "matchstick man" simplification method and "volume simplification" method. Though both of these two methods are convenient and practical for people to understand the overall shape of human body, they seldom involve the performance study of certain piece of muscle. Based on microscopic view, this paper would discuss how to simplify one piece of muscle, hoping to give some enlightenment to people who are engaged with teaching or learning of human anatomy for artists and help them get a better understanding.

\section{ANALYSIS ON "SIMPLIFICATION"}

Gombrich wrote in his Art and Illusion that the filing method of our mind is totally different from the measuring system in science and that things which are different objectively may looks similar while things which are similar objectively may looks entirely different. Generally speaking, painting is just an expression of image, which has already existed in human being's mind. Thus people tend to simplify the image when painting. The definition of simplification is as follows: according to Art and Visual Perception wrote by Arnheim, I have concluded two levels of meaning for the simplification. One is laying emphasis on the impact and role of objective existence on observer; the other one is limitation on the scope of subjective reflection by the subject. Kurt Beidirt simplified the definition of art as the wisest organization method people could acquire on the basis of understanding the nature. The nature means that all things are subject to their own nature. The feasibility of simplification: not only human beings' psychological activity follows the rule of simplification, but also the objective world follows the rule of simplification. It is the subjective condition for simplification that human beings' psychological activity follows the rule of simplification. When Arnheim was explaining the concept of consciousness, he said from the very beginning people's perception has the tendency of grasping rough features of structure. That the outside world follows the rule of simplification constitutes the objective condition of simplification. Max Wertheimer, the founder of Gestalt psychology, thought that the consistency between perceptual structure and physical structure is caused by the adaptation, which refers to the nervous system in the evolutionary system adapt to the surroundings. Thus, we may add "separation is simplification" to "manifestation is separation" proposed by Goethe, that is, "manifestation is separation and separation is simplification". The nature of "manifestation" is to separate things from surroundings, and "separation" on visual perception actually refers to the "simplification". The method of simplification: we could use the amount of structural features, which constitute a piece of muscle, to explain the simplification. In some absolute sense, when one 
piece of muscle has only several structural features, it would reach the simplification. In a relative sense, if the complicated muscle was made up into an orderly overall by a simple form with structural features as little as possible, we may say that it was simplified, just as the simplification expressed in Picasso and His Bull.

\section{THE SIMPLIFICATION OF HUMAN ANATOMY FOR ARTISTS IN THE TEACHING ACTIVITIES}

In order to give a brief introduction of the theoretical contents of simplification, I will introduce the simplification with my experience, which I have used and accumulated in the teaching practice. It will be discussed in the following order: from the head and neck and facial features to the body and then the four limbs.

\section{A. Head and Neck and facial features:}

Eyeball, almost having the similar size with table tennis, is a human organ exposed to the outside world and is protected by eyebrow bone and cheekbone, as well as the upper eyelid in large radian and the lower eyelid in small radian. We may regard the eyelid as short visor covering the eye, or goggle for the eyeball; looking from the side of eye, it would be clearly that the upper and lower eyelids form a 45 degree angle. Due to different radians, the upper and lower eyelids form a rhombus. Nose Tip: When you are painting a nose, you can regard the nose tip and wings of nose as three circles. What you need to do is paying attention to the forth and back coverage ratio and size ratio. Mouth: The mouth is praised as Cupia's Bow. The upper lip can be simplified as a flat "M". The upper lip in "M" shape has a fovea, also called philtrum, protruding like the head of boat. This fovea is tubercle. The lower lip can be simplified as a flat "W". Both sides of "W" form protruding surface. Ear: ear is composed by outer ear round, inner ear round, tragus, antitragus, lobulus auriculae, triangular fossa. We may simplify the outer ear round as "C" or a hook; the inner ear round could be simplified as "Y", with the middle cross of "Y" as triangular fossa. With such simple shapes, it would be easy for people to sketch-up and mould. Eyebrow: there are different types of eyebrows, including arched eyebrows, flat eyebrows, triangle eyebrows, "乙"shape eyebrows, dashing eyebrows, eight-character eyebrows, crescent eyebrows, bending water eyebrows. From all these types, we may see people's simplified understanding of eyebrows. The two clavicles, on the whole, looks like a hanger. When separated, each clavicle looks like a stretched "S".

\section{B. Major muscle of body:}

Trapezius muscle extends longitudinally from the occipital bone to the lower 12th thoracic vertebrae and laterally to the spine of the scapula (shoulder blade). Function: Triceps work together to raise the shoulder. The shape of trapezius can be regarded as dagger; Though trapezius belongs to dorsal muscle on the trunk, it is at the top of shoulders. From the front, we can see trapezius' influence on the outline of neck. Trapezius and clavicle together can be simplified as a hanger, which begins from shoulder and ends at clavicle and connects with the upper 7th cervical vertebra. Deltoid muscle always looks like a triangle, no matter from the side or the front, only a little different on the angle. Pectoralis major arises from the anterior surface of the sternal half of the clavicle; from breadth of the half of the anterior surface of the sternum, as low down as the attachment of the cartilage of the sixth rib. The pectoralis major is responsible for keeping the humerus attached to the trunk of the body. As it covers from the 1st to the 6th rib, it has 6-9 muscle articles, showing the shape of pentagon. The outline and organization structure of pectoralis major is fanshaped divergent state. We can simplify the outline of pectoralis major as a fan placed on the thorax, with the handle of fan as the muscle connected to humerus. In this way, it will be easy to describe the muscle articles. Women's breast, attached on the pectoralis major, can be regarded as a comma, pointing to the lower arm. There is another method to understand the pectoralis major. That is interpreting the pectoralis major as a fish without head, on which we can add a circle as the breast. In this way, we can change the shape of breast. Musculus serratus anterior originates on the surface of the 1 st to 8 th ribs at the side of the chest and inserts the downside border of the scapula. Musculus serratus anterior can pull the scapula forward and lift the shoulders and arms. The figure of Musculus serratus anterior can be understood as structure of a ball. Its outline can be simplified as a banana attached on the chest. The shape of rectus abdominis can be simplified as a bullet. Its curved part can be put into pelvis and lower part ends at the joint of pubis and upper part which is flat attaches to the rib. The shape of rectus abdominis can be simplified as eight parts, which are bilateral symmetry. When modeling, one should pay attention to the difference of rectus abdominis between man and woman. The dividing lines of eight parts of rectus abdominis are usually painted on a horizontal direction. Actually they are on an angle. Dorsal muscle groups on the trunk can be represented by "M", which will be easy to understand. The capital "M" should be placed at the back of rib, under the shoulders and till the bottom of scapula. Two vertical lines of "M" represent the edge of scapula. Thus, the scapula is settled. The deep " $\mathrm{V}$ " in the " $\mathrm{M}$ " is line of trapezius muscle. Thus, trapezius muscle is settled. The side latissimus dorsi originates from the bottom of two vertical lines of "M" to "V". The middle and lower parts of muscles include multifidus muscle and external oblique, which extend outward from the lowest point of "V". Identifying muscle group in this way will help us understand body's movement. It will easy to identify and memorize the relative position of trunk dorsal muscle. The latissimus dorsi start from the 7 th thoracic vertebra of the spine and extend to the sacrum and end at the inside top of humerus. It is responsible for the downward and backward movement of the upper arm. In the Figure Drawing Design and Invention, Michael Hampton wrote that the shape of the latissimus resembles a bowl with arms and legs and the arms on the side of the bowl are small triangles 1 . When the textbook compiling group from Zhejiang Academy of Art is writing the book Body Structure, they simplified the back trunk as a

\footnotetext{
${ }^{1}$ Figure Drawing design and invention P116 "Hollis CooperThe shape of the latissimus resembles a bowl with arms and legs. The arms on the side of the bowl are small triangles. The design of these arms suggests that the shape of the latissimus, like the pectoralis, unflods when it is stretched."
} 
goblet. The top edge of the goblet keep in line with the upper edge of shoulder blade, while the goblet body is the outline of latissimus dorsi. The base of goblet is the lower edge of the hips. In the practice, I have found that the outline of latissimus dorsi and trunk and pelvis can be simplified as a Coke bottle. Split the label symmetrically, and then cut off a part, so as to make it a triangle. This triangle can be regarded as the point where the trapezius stops at the upper edge of humerus. 2 When painting the buttock, the pelvic muscle looks like a butterfly from the behind. The upper wings are the gluteus medius under the pelvic curve. The lower wings are gluteus maximus, or the gluteus. At the separating point of the upper and lower wings, the surface of greater trochanter of femur appears.

\section{Four Limbs:}

The structure of muscle on the arms can be simplified as the chain, which is interlocking. They are also similar on functions. When viewed from the front, the shape of leg can be simplified as a stretched "B" with the lower part becoming smaller. The straight line of " $B$ " control the inside outline of leg. The outside outline of leg becomes smaller towards the knee and ankle. The back leg appearance has the similar rule with the front view. We can use a simplified "B" to describe a leg with protruding ankle. We may use an inversed " $B$ " to represents the three-quarter of back leg. The side view of leg can be simplified as the alphabet " $\mathrm{S}$ " with the thigh curve representing the bulging muscle on the front of thigh and the crus curve representing the protruding gastrocnemius muscle on the back of crus. The shape of kneecap can be simplified as watch with soft belt. The iliotibial tract on the side of leg, the insert point for tensor fasciae latae, is attached to vastus lateralis muscle and finally insert at the tibia. As for the shape of iliotibial tract, we may simplify it as a spanner on the caput femoris. The bottom of the spanner, handle of spanner, extent downward to crus. Its work method is similar with the thigh. We may simplify the joint from the crus to anklebone as a spanner that closely grasp the ball. The spanner refers to the end of crus, while the ball represents the upper bone of the ankle. In order to better reflect the function of foot, we could put a needle through the above two parts. If the spanner is attached to the ball, this ball could only move forward or backward or rotate. Because these two parts are linked together by a needle. Based on the structural composition of foot bone, we may regard, from different views, the foot as an arch or "C" shape. From the side view, the foot, from the front to the back, looks like an arch or "C" rotated clockwise 90 degrees. And this " $\mathrm{C}$ " is stretched. From the front view, the foot, from one side to the other side, also looks like a " $C$ ", which is also rotated clockwise 90 degrees. Three quarters of foot side will be the combination of the above two simplified shapes. Such simplification method will help us understand the character

\footnotetext{
2 The reason why there are differences on simplification: one reason is that one certain piece of muscle existed in human being's body. The other reason is that people have original schemes of experience, that is to say, one certain piece of muscle remains the same, while the schemes of experience existed in observers varied greatly. Thus it is normal to have different simplified outcomes. As long as the structure of muscle and the simplified symbol keep consistent, such consistence will be "isomorphism" in Gestalt Psychology.
}

and function of foot. With the above simplified shape and function, when you are painting the foot, you can simplify the foot as triangle from most visual angles. This method can not only draw simple actions, but also make the weight sense of foot easy to portray. There is no need to pay much attention to the outline or details of foot. Image the shape of foot with socks. What's more, the load-bearing foot is similar to the triangle, which is stable on the vision. The foot has four piece of muscle padding, including (1) heel (2) foot ectoloph padding ( abductor, originating from the heel and ends at the little toe ) (3) "big padding muscle "( under four toes ) (4) padding muscle under the big toe. We may generalize the four piece of basic padding muscles as circles or ovals. And the shape of combination of heel and foot ectoloph padding is like a comma.

\section{Summary:}

According to the above discussion on simplification, we may get three types of simplification: the first one is alphabets; the second one is simple figures and symbols; the third one is daily items.

1) Alphabets: such as $M W C Y S M V B S C$. in summary, all the above alphabets are the transformation and combination of the alphabet " $C$ ". For instance, " $\mathrm{M}$ " is the combination of two inversed "c". Turn over " $M$ ", we will get "W". "V" is the transformation of "C". "B" looks likeb, and "C" looks likes. The outer convex structure of " $\mathrm{C}$ " verifies the saying by Mattise, "any body's shape is outer convex and you can never find concave line on the body."

2) Basic figures and symbols: such as circle, triangle, rectangle and so on. I would take circle as an example to show human beings usually give priority to such basic shapes. Just as Gombrich wrote in his Art and Illusion, "the vision gives priority to circles, which is also under the rule of simplification. The circle, which is central symmetry, doesn't emphasize any direction. We may say it is the simplest visual style...... This shows that human being's vision has a great love towards the circle."

3) In this paper, I simplify muscles as dagger, inversed hanger, folding fan, banana, goblet, chain, butterfly, watch with soft belt and spanner. The reason why I can summarize such forms is that I have conformed to the relations between the form and function. It will help us to understand.

\section{The MEANING OF SIMPLIFICATION}

Arnheim wrote in his Art and Vision Perception, "what the cognitive competence looks for is the order. The mission of science is to extract the well-regulated orders from diverse phenomena, while the mission of art is to show the well-regulated orders existing in such diverse phenomena through images." And simplification is the most effective method to show the orders. Only when human beings' ability of grasping simplification, which belongs to the reason, gets developed, can we fine the orders in the nature. 


\section{REFERENCE:}

[1] Zhejiang Academy of Fine Arts, Body Structure, People's Fine Arts Publishing House, Beijing, 1978

[2] Burne Hogarth. Dynamic Figure Drawing, Guangxi Fine Art Press, Guiling, 1997

[3] Sun tao, Ye nan. Deconstruction: Anatomy for Artists, People's Fine Arts Publishing House, Beijing, 2005

[4] Hollis Cooper, Figure Drawing : Design and Invention, 2009

[5] Xu jing, Liu jian, Human body and Animal structure, Jiangxi Fine arts Press, Nanchang, 2011

[6] George Bridgman. Bridgman's Complete Guide to Drawing from Life, Guangxi Fine Art Press, Guiling, 2012

[7] Victor Perard. Anatomy and Drawing, Dover Publications Inc, 1989

[8] Sarah Simblet. Anatomy for Artists, Zhejiang Photography Press, Hangzhou, 2004

[9] Arnheim. Art and Visual Perception, Chinese Social Science Press, Beijing, 1984

[10] Gombrich. Art and Illusion, Hunan science and Technology Press, Changsha, 1997 